\title{
Electrical conductivity and Hall conductivity of a hot and dense quark gluon plasma in a magnetic field: A quasiparticle approach
}

\author{
Arpan Das, ${ }^{1, *}$ Hiranmaya Mishra, ${ }^{1, \dagger}$ and Ranjita K. Mohapatra ${ }^{2,3, *}$ \\ ${ }^{1}$ Theory Division, Physical Research Laboratory, Navrangpura, Ahmedabad 380 009, India \\ ${ }^{2}$ Department of Physics, Indian Institute of Technology Bombay, Mumbai 400076, India \\ ${ }^{3}$ Department of Physics, Banki Autonomous College, Cuttack 754008, India
}

(Received 20 July 2019; revised manuscript received 11 January 2020; accepted 3 February 2020; published 24 February 2020)

\begin{abstract}
We estimate here the electrical and Hall conductivity using a quasiparticle approach for quark matter. We use a Boltzmann kinetic approach in the presence of external magnetic field. We confront the results of model calculations with lattice QCD simulations for vanishing magnetic field. In general, electrical conductivity decreases with magnetic field. The Hall conductivity on the other hand can show a nonmonotonic behavior with magnetic field due to an intricate interplay of behavior of relaxation time and strength of the magnetic field. We argue for vanishing quark chemical potential Hall conductivity vanishes and quark gluon plasma with finite quark chemical potential can show Hall effect. Both electrical conductivity and Hall conductivity increase with increasing quark chemical potential.
\end{abstract}

DOI: 10.1103/PhysRevD.101.034027

\section{INTRODUCTION}

Relativistic heavy-ion collision experiments at Relativistic Heavy Ion Collider (RHIC) and Large Hadron Collider (LHC) provide an opportunity for a comprehensive understanding of quantum chromodynamics (QCD) in nonperturbative regime, especially for the phase diagram of strongly interacting matter. Large number of experimental data, as well as theoretical models, gives ample evidence of the formation and subsequent evolution of a deconfined strongly interacting matter, known as quark gluon plasma (QGP). It is expected that strongly interacting QCD plasma achieves local thermal equilibrium within about a $1 \mathrm{fm}$ time. For a comprehensive and detailed understanding of the hot and dense QCD medium, transport coefficients, e.g., shear viscosity, bulk viscosity, electrical conductivity, are very important. These coefficients enter as essential theoretical input for the hydrodynamical simulations which are important tools for interpreting heavy-ion collision data. A small shear viscosity to entropy ratio $(\eta / s)$ is consistent with the transverse momentum spectra of the charged particles within the framework of dissipative hydrodynamical model of quark gluon plasma [1-3]. $\eta / s$ of the strongly interacting

\footnotetext{
*arpan@prl.res.in

†hm@prl.res.in

"ranjita.iop@gmail.com
}

Published by the American Physical Society under the terms of the Creative Commons Attribution 4.0 International license. Further distribution of this work must maintain attribution to the author(s) and the published article's title, journal citation, and DOI. Funded by SCOAP. plasma also satisfies the lower bound of shear viscosity to entropy ratio, $\eta / s=\frac{1}{4 \pi}$, obtained using gauge gravity duality (AdS/CFT correspondence) [3]. This apart bulk viscosity $\zeta$ also plays an important role in the relativistic dissipative hydrodynamics describing the evolution of quark gluon plasma [4-12]. First principle lattice Monte Carlo simulations show a nonmonotonic behavior of $\zeta / s$ as well as $\eta / s$ near the critical temperature $T_{c}$. [6-12].

Further, plausibility of a generation of strong magnetic field in noncentral heavy-ion collision experiments brings novel phenomenological aspects. The strength of the magnetic field so produced strongly depends upon the center of mass energy of the collision. In fact, at RHIC energies, the strength of the magnetic field is expected to be as large as $e B \sim$ few $m_{\pi}^{2}$ and at LHC energies it can go even higher, of the order of $e B \sim 15 m_{\pi}^{2}$, at least in the initial stage $[13,14]$. Although the strength of the magnetic field is large at the initial stage in these collisions, strength of the magnetic field is expected to decay with time. Decay of the electromagnetic field in the absence of conducting medium is fast, but due to finite electrical conductivity $\left(\sigma^{\mathrm{el}}\right)$ of the plasma, the strength of the magnetic field can be nonvanishing in QGP. Nonvanishing magnetic field can affect significantly the evolution of the strong interacting matter [15-33]. This apart nonvanishing magnetic field along with the topologically nontrivial non-Abelian QCD vacuum can give rise to novel charge conjugation and parity $(\mathrm{CP})$ violating effects such as chiral magnetic effect and chiral vortical effect, etc. [34]. To estimate various transport coefficients of QGP and subsequent hadronic medium, different complementary approaches, e.g., perturbative QCD, QCD inspired effective models, etc. have been investigated in literature 
[18-33,35-63]. Apart from the viscosity coefficients, the conductivities both electrical and thermal conductivity also play an important role in the hydrodynamical evolution of strongly interacting medium at nonzero baryon densities $[64,65]$. Recently, the thermoelectric effect of the hadronic medium produced at the later stages of heavy-ion collisions has been investigated within the framework of hadron resonance gas model [63].

In the present work, we investigate electrical conductivity as well as Hall conductivity of quark gluon plasma produced in heavy-ion collisions. The Hall effect in a conducting medium is the manifestation of the generation of an induced electric current transverse to an electric field and magnetic field (perpendicular to the electric field). Collision geometry in heavy-ion collisions can give rise to configuration where electric field and magnetic field are transverse to each other $[66,67]$. Hence, it is interesting to study the Hall effect for the electrically conducting QCD medium produced in heavy-ion collisions. Hall conductivity of QGP has been studied within the framework of perturbative QCD in Ref. [68]. Recently, we studied Hall conductivity of the hadronic medium within the framework of hadron resonance gas model [69]. In the present investigation, we estimate the electrical and Hall conductivity for the hot and dense QGP in a magnetic field using quasiparticle picture of quark gluon plasma. It is important to note that QGP can be approximated as an ideal gas of quarks and gluons only at asymptotic high temperatures. However, lattice thermodynamics results indicate that, for the temperature range achievable at RHIC and LHC, QGP cannot be approximated as an ideal gas of quarks and gluons, i.e., the Steffan Boltzmann's limit for an ideal gas cannot be achieved at RHIC and LHC. Further, the plasma produced in the heavy-ion collision is a strongly coupled liquid rather than a weakly coupled ideal gas. Particularly, near the QCD transition temperature, nonperturbative effects are important. Only first principle lattice QCD calculations are a reliable way to study the thermodynamics and transport properties of the strongly interacting system. However, for a phenomenological reason, quasiparticle models are constructed. The parameters of these quasiparticle models are fixed by comparing thermodynamic results obtained in these models with lattice QCD results [70-90]. We have used in this investigation two such thermodynamically consistent well-explored quasiparticle models to estimate electrical conductivity and Hall conductivity in the presence of a magnetic field. Our results indicate that although the quasiparticle models considered in this work are thermodynamically on the same footing, but in the context of transport coefficients, they are different. For the validity of our results obtained using these quasiparticle models, we have also given a comparison of our results with the lattice QCD results wherever available.

Generically in quasiparticle picture, nonperturbative dynamics is encoded in the masses of quasiparticles. The masses of the particles can be imagined to be arising from the energy contained in a strongly coupled volume determined by the correlation range of the interaction. Once the effect of interaction is taken care of this way, the quasiparticles behave like free gas of massive constituents [70-79]. Instead of medium-dependent mass, one can also consider the quasiparticle picture by introducing effective fugacity parameter in the distribution function [88]. This effective fugacity does not change the mass of the particles rather it changes the single particle dispersion relation. It is important to note that in low energy condensed matter systems with specific type of charge carriers (either electrons or holes), e.g., semiconductors etc., show Hall effect [91]. Similarly, an electron-ion plasma also shows Hall effect, because in electron-ion plasma mobility of electrons and ions is different. Hence, a net Hall current exists in electron-ion plasma. However, for pair plasma (e.g., electron positron plasma) due to vanishing net gyration frequency of the charge carriers net Hall current vanishes [92-94]. Quark gluon plasma at vanishing baryon chemical potential is analogous to the case of pair plasma. Hence, QGP at vanishing quark chemical potential does not show Hall effect due to the exact cancellation of Hall current due to particles and their antiparticles. However, at finite baryon chemical potential, numbers of positive and negative charge carriers are not same, due to asymmetry between the numbers of baryons and antibaryons. Hence, electrically charged quark gluon plasma with net baryon number, expected to be produced at low energy collisions, will also show Hall effect $[95,96]$.

Keeping the above motivation in mind, we calculate the electrical conductivity and Hall conductivity of quark gluon plasma in a magnetic field within the kinetic theory framework using relaxation time approximation. In Ref. [68], electrical conductivity and Hall conductivity have been estimated using perturbative QCD approach. However, QGP formed in heavy-ion collision experiments is strongly coupled. Hence, perturbative approach may not be sufficient to study the transport coefficients of quark gluon plasma. In this context, quasiparticle model of QGP, where quasiparticle nature of the particles encodes the nonperturbative effects, has been used to study various transport coefficients [21,97-105]. In this investigation, we estimate the electrical conductivity and Hall conductivity in a magnetic field using quasiparticle picture of QGP. In the initial stage of the heavy-ion collision, the strength of the magnetic field is very large compared to the QCD scale $\left(\Lambda_{\mathrm{QCD}}\right)$. But what fraction of this magnetic field can survive in the strongly interacting medium is not a settled issue, and the strength of the magnetic field in the plasma is intimately connected with the value of electrical conductivity. In fact, for magnetohydrodynamic modeling of the strongly interacting medium, the electrical conductivity usually is taken to be infinity, which is far from the practical situation $[15,16]$. Due to finite and small electrical conductivity, only 
a fraction of the initial large magnetic field can survive in the thermalized strongly interacting medium.

As mentioned earlier, we attempt to calculate the electrical conductivity and Hall conductivity in the presence of magnetic field using the relaxation time approximation in Boltzmann kinetic equation. In this context, it may be noted that the thermalization of the strongly interacting medium is governed by QCD processes. In this thermalization process, gluons play a dominant role, because the initial density of the gluons is large with respect to the quarks and antiquarks. Magnetic field does not affect directly the interaction between gluons. Only quarks and antiquarks are affected by the magnetic field. Since in the thermalization of the strongly interacting medium, gluons play a dominant role, it is expected that the effect of the magnetic field should be subdominant. In fact, if we take the initial temperature of the strongly interacting matter to be of the order of $T \sim 2-3 T_{c}$, then $T^{2}>q B$ where $B \sim m_{\pi}^{2}$. Hence, temperature can be the dominant scale in QGP. Of course, if one is interested in the case where $q B \gg T^{2}$, then the magnetic field becomes the dominant scale and, in that case, one needs to consider the effect of the magnetic field on the distribution function and collision integral of the Boltzmann equation. Again, in the hadronic medium, the magnetic field should be even smaller than that of QGP. Hence, if we consider the magnetic field to be subdominant scale, which is quite reasonable, then the relaxation time approximation can be used as the simplest approximation of the collision integral to solve the Boltzmann equation and this approximation in the presence of magnetic field for QGP has been used in Refs. [68,106-110], etc. However, to capture the effect of the magnetic field in its full glory, one should consider general collision integral with magnetic field-dependent scattering matrix, which however is beyond the scope of this work. More general collision term including the effect of magnetic field in the scattering cross section as well as in the phase space has been considered in the strong magnetic field limit in Refs. [111,112]. Hence, in this investigation, we assume thermalization is governed by the strong interaction and the phase space and the single particle energies are not affected by magnetic field through Landau quantization [68]. Effect of magnetic field only enters in the calculation through the cyclotron frequency of the charged particles. In this investigation, we consider that the thermalization of the strongly interacting medium is achieved due to the strong interaction and the external magnetic field which is generically relatively small with respect to the dominant scale of the system. Hence, external field only produces a small deviation of the system from the equilibrium.

This paper is organized as follows: in Sec. II, we briefly summarize the formalism to estimate electrical conductivity and Hall conductivity using kinetic theory within relaxation time approximation as given in Refs. [68,69]. In Sec. III, we briefly discuss the quasiparticle models of quark gluon plasma considered in this work. In Sec. IV, we present and discuss the results for electrical and Hall conductivity. Finally, we summarize our work with an outlook in the conclusion section.

\section{BOLTZMANN EQUATION IN RELAXATION TIME APPROXIMATION}

In the presence of external electromagnetic field, the relativistic Boltzmann transport equation for a particle with electric charge $e$ can be written as [68]

$$
p^{\mu} \partial_{\mu} f(x, p)+e F^{\mu \nu} p_{\nu} \frac{\partial f(x, p)}{\partial p^{\mu}}=\mathcal{C}[f],
$$

where the electromagnetic field strength tensor is denoted as $F^{\mu \nu}$. On the right-hand side of Eq. (1), $\mathcal{C}[f]$ represents the collision integral which in the relaxation time approximation can be written as

$$
\mathcal{C}[f] \simeq-\frac{p^{\mu} u_{\mu}}{\tau}\left(f-f_{0}\right) \equiv-\frac{p^{\mu} u_{\mu}}{\tau} \delta f,
$$

where $u_{\mu}$ is the fluid four velocity and in the local rest frame it has the form, $u_{\mu} \equiv(1, \overrightarrow{0})$. In Eq. (2), $\tau$ is the relaxation time. At this point, it is important to emphasize that in general relaxation time $(\tau)$ depends on the scattering cross section and the number density of the particles. The scattering cross section is a function of the momenta and energy of the particles. One could use energy and momenta-dependent relaxation time to solve the Boltzmann equation. However, for simplicity in the relaxation time approximation, one uses the relaxation time averaged over the distribution function of the particles in thermal equilibrium. Hence, the thermal averaged relaxation time is independent of the energy and momentum. However, temperature and chemical potential dependence come through the equilibrium distribution function. In the present investigation, we consider thermal averaged relaxation time. Relaxation time determines the time scale over which a nonequilibrium system relaxes toward its equilibrium state in the presence of small external perturbation. The equilibrium state of the system is characterized by the equilibrium distribution function $f_{0} . f$ represents out of equilibrium distribution function. The underlying assumption of the relaxation time approximation is that the external perturbation takes the system slightly away from equilibrium and then it relaxes toward equilibrium, exponentially with a time scale $\tau$. In this approximation, the external perturbation, which in this case is external electromagnetic field, not dominant scale with respect to the characteristic scale of the thermal system in equilibrium. Hence, we are not considering the effect of Landau quantization on the phase space of the particles and in the scattering processes. The equilibrium distribution function satisfies $\left(f_{0}\right)$, 


$$
\begin{aligned}
\frac{\partial f_{0}}{\partial \vec{p}} & =\vec{v} \frac{\partial f_{0}}{\partial \epsilon}, \quad \frac{\partial f_{0}}{\partial \epsilon}=-\beta f_{0}\left(1-f_{0}\right), \\
f_{0} & =\frac{1}{1+e^{\beta(\epsilon \pm \mu)}},
\end{aligned}
$$

where $\epsilon(p)=\sqrt{\vec{p}^{2}+m^{2}}$ is the single particle energy, $\mu$ is the quark chemical potential, and $\beta=1 / T$ is the inverse of temperature. Using Eq. (2), the Boltzmann equation (1) can be written in the following manner $[68,69]$ :

$$
\frac{\partial f}{\partial t}+\vec{v} \cdot \frac{\partial f}{\partial \vec{r}}+e[\vec{E}+\vec{v} \times \vec{B}] \cdot \frac{\partial f}{\partial \vec{p}}=-\nu\left(f-f_{0}\right),
$$

where $\nu=1 / \tau$ is the inverse of relaxation time. In case of uniform and static medium, where $f$ and $f_{0}$ are independent of time and space [68], Eq. (4) simplifies to

$$
-e[\vec{E}+\vec{v} \times \vec{B}] \frac{\partial f}{\partial \vec{p}}=\nu\left(f-f_{0}\right) .
$$

Here without loss of generality, electric field and magnetic field transverse to each other and can be chosen in the following way: $\vec{E}=E \hat{x}$ and $\vec{B}=B \hat{z}$. For this representation of $\vec{E}$ and magnetic field $\vec{B}$, the out of equilibrium distribution function which is a solution of Eq. (5) can be shown to be [69]

$f(p)=f_{0}-e E v_{x}\left(\frac{\partial f_{0}}{\partial \epsilon}\right) \frac{\nu}{\nu^{2}+\omega_{c}^{2}}+e E v_{y}\left(\frac{\partial f_{0}}{\partial \epsilon}\right) \frac{\omega_{c}}{\omega_{c}^{2}+\nu^{2}}$.

Here $\omega_{c} \equiv(e B) /(\varepsilon(p))$ is the cyclotron frequency.

Electric current can be defined in the following way [68]:

$j^{i}=e \int \frac{d^{3} p}{(2 \pi)^{3}} v^{i} \delta f=\sigma^{i j} E_{j}=\sigma^{\mathrm{el}} \delta^{i j} E_{j}+\sigma^{H} \epsilon^{i j} E_{j}$,

where $\epsilon_{i j}$ is the antisymmetric $2 \times 2$ unity tensor, with $\epsilon_{12}=-\epsilon_{21}=1$. Using Eqs. (6) and (7), the electrical and the Hall conductivity for a system of multiple charge particle species can be expressed as $[68,69]$

$\sigma^{\mathrm{el}}=\sum_{i} \frac{e_{i}^{2} \tau_{i} g_{i}}{3 T} \int \frac{d^{3} p}{(2 \pi)^{3}} \frac{p^{2}}{\epsilon_{i}^{2}} \frac{1}{1+\left(\omega_{c i} \tau_{i}\right)^{2}} f_{0}\left(1-f_{0}\right)$,

$\sigma^{H}=\sum_{i} \frac{e_{i}^{2} \tau_{i} g_{i}}{3 T} \int \frac{d^{3} p}{(2 \pi)^{3}} \frac{p^{2}}{\epsilon_{i}^{2}} \frac{\omega_{c i} \tau_{i}}{1+\left(\omega_{c i} \tau_{i}\right)^{2}} f_{0}\left(1-f_{0}\right)$,

where $e_{i}, \tau_{i}, g_{i}$, and $\omega_{c i}$ are electric charge, thermal averaged relaxation time, degeneracy factor, and cyclotron frequency of the $i$ th charged particle species, respectively. It is important to note that in the absence of magnetic field, Eq. (8) reproduces the standard expression of electrical conductivity in relaxation time approximation [21,113].
It is easy to see from Eqs. (8) and (9) that particles and their antiparticles contribute to the electrical conductivity in a same manner and their behavior is opposite in case of Hall conductivity. Using quasiparticle picture of quark gluon plasma, one can get relaxation time $(\tau)$, medium-dependent mass $(m)$, as well as the medium-dependent dispersion relation. Once these quantities are known using Eqs. (8) and (9), electrical conductivity and the Hall conductivity can be estimated.

At this point, it is important to emphasize that in this investigation we have considered only magnetic field and the electric field perpendicular to each other; however, for a more general configuration of electric and magnetic field, the electrical conductivity tensor can be expressed as [114]

$$
\sigma_{i j}=\delta_{i j} \sigma_{0}-\epsilon_{i j m} h_{m} \sigma_{1}+h_{i} h_{j} \sigma_{2},
$$

where $\vec{h}=\frac{\vec{B}}{|\vec{B}|}$ and

$\sigma_{n}=\frac{e^{2}}{3 T} \int \frac{d^{3} p}{(2 \pi)^{3}} \tau\left(\frac{p^{2}}{\epsilon^{2}}\right) \frac{\left(\omega_{c} \tau\right)^{n}}{1+\left(\omega_{c} \tau\right)^{2}} f_{0}\left(1-f_{0}\right), \quad n=0,1,2$

Hence, for a general configuration of electric and magnetic field, the electrical conductivity tensor has three components $\sigma_{0}, \sigma_{1}$, and $\sigma_{2} . \sigma_{1}$ and $\sigma_{2}$ are associated with $\vec{E} \times \vec{B}$ and $\vec{E} \cdot \vec{B}$ terms in the electrical conductivity tensor. However, when the electric and the magnetic field are perpendicular to each other, we only have $\sigma_{0}$ and $\sigma_{1} . \sigma_{0}$ can be identified with the electrical conductivity in the presence of a magnetic field $\left(\sigma^{\mathrm{el}}\right)$, and $\sigma_{1}$ can be identified as the Hall conductivity $\sigma^{H}$. $\sigma_{2}$ is nonvanishing when the electrical and magnetic field are not perpendicular to each other. If we consider the magnetic field along the $z$ direction, then the electrical conductivity along the $z$ direction, $\sigma_{z z}=\sigma_{0}+\sigma_{2}$, which is independent of the magnetic field as can be seen from the explicit expressions of $\sigma_{0}$ and $\sigma_{2}$. However, $\sigma_{1}$, the Hall conductivity, is perpendicular to the magnetic field.

Let us note that in Refs. $[115,116]$ transport coefficients have also been calculated using a 14-moment approximation for dissipative magnetohydrodynamics. In these studies, the effects of Landau quantization have not been considered. As is the case in the present work, the effect of Landau quantization is not included and further more number of transport coefficients arise in the presence of magnetic field with respect to zero magnetic field case. Comparison of our analysis with Refs. $[115,116]$ is in order here. For a single species, the Hall conductivity can be expressed as

$$
\sigma^{H}=\frac{e^{2} \tau}{3 T} \int \frac{d^{3} p}{(2 \pi)^{3}} \frac{p^{2}}{\epsilon^{2}} \frac{\omega_{c} \tau}{1+\left(\omega_{c} \tau\right)^{2}} f_{0}\left(1-f_{0}\right) .
$$


In the Boltzmann approximation, for massless case with $\tau=\lambda_{\mathrm{mfp}}$,

$$
\begin{aligned}
\sigma^{H} & =\frac{e^{2} \tau}{3 T} \int \frac{d^{3} p}{(2 \pi)^{3}} \frac{\omega_{c} \tau}{1+\left(\omega_{c} \tau\right)^{2}} f_{0} \\
& =\frac{e^{2} \tau}{3 T} \int \frac{d^{3} p}{(2 \pi)^{3}} \frac{\xi_{B} / 3}{1+\left(\xi_{B} / 3\right)^{2}} f_{0} \\
& =\frac{e^{2} \lambda_{\operatorname{mfp}}}{3 T} \frac{3 \xi_{B}}{9+\xi_{B}^{2}} n
\end{aligned}
$$

where $\xi_{B}=\frac{e B \lambda_{\mathrm{mfp}}}{T}$. To derive this, we have replaced $\omega_{c} \tau \equiv$ $\frac{e B \tau}{\epsilon(p)}$ by its thermal averaged value, i.e., $\frac{e B \tau}{\bar{\epsilon}} \equiv \frac{1}{3} \xi_{B}$, where $\bar{\epsilon}=3 T$, the average single particle energy at a given temperature. For $\xi_{B} \gg 1, \sigma^{H}$ is independent of $\lambda_{\mathrm{mfp}}$. WiedemannFranz law connects electrical conductivity with the heat-diffusion coefficient. For massless Boltzmann gas in the presence of magnetic field and for $\xi_{B} \gg 1$, it has been shown in Refs. $[115,116]$ that the Hall type heat-diffusion coefficient is independent of the mean free path. Due to Wiedemann Franz law, this also means that the Hall conductivity is also independent of the mean free path for $\xi_{B} \gg 1$. This is consistent with the formalism considered in this investigation.

\section{QUASIPARTICLE MODEL OF QUARK GLUON PLASMA}

To describe the thermal properties of QGP, one uses QCD at finite temperature and baryon chemical potential. At very high temperature, due to asymptotic freedom, a system of quarks and gluons can be treated as ideal gas. But at relatively low temperature, near $T_{c}$, nonperturbative effects become important. In the nonperturbative domain, first principle lattice calculations give reliable prediction about different thermal properties of the system. However, for phenomenological aspects, one needs an effective description of QGP near $T \sim T_{c}$. In this context, one can use quasiparticle description of quarks and gluons in medium to investigate thermal properties of QGP. The basic idea behind various quasiparticle pictures of QGP is that one can approximate the thermodynamic properties of a system of strongly interacting quarks and gluon by a system of quasi quarks and quasi gluons, where the information about the interaction is encoded in the physical properties, e.g., medium-dependent mass of the quasiparticles. In literature, various types of quasiparticle models are discussed [70-90]. In this investigation, we have considered two quasiparticle models, quasiparticle model I (QPM I) and quasiparticle model II (QPM II). In QPM I, quark gluon plasma is described by an ideal gas of quasiparticles having temperature-dependent mass arising from the interactions with the surrounding quarks and gluons in the medium [81-83]. In QPM II, we consider the effective fugacity quasiparticle model, where the quasiparticle nature is implemented by modifying the distribution function for free quarks and gluons by introducing an effective fugacity, which encodes information of the interaction [88].

\section{A. Quasiparticle model I (QPM I)}

In this quasiparticle model, effective mass of all the quark (antiquark) has bare mass $\left(m_{0}\right)$ as well as thermal mass $\left(m_{\text {th }}\right)$, which can be expressed as [81-83]

$$
m^{2}=m_{0}^{2}+\sqrt{2} m_{0} m_{\mathrm{th}}+m_{\mathrm{th}}^{2} .
$$

The thermal mass $\left(m_{\mathrm{th}}\right)$ arises due to the interaction of quarks and antiquarks with the other constituents of the plasma and can be expressed as [117]

$$
m_{\mathrm{th}}^{2}(T, \mu)=g^{2}(T, \mu) T^{2} \frac{N_{c}^{2}-1}{8 N_{c}}\left(1+\frac{\mu^{2}}{\pi^{2} T^{2}}\right),
$$

where $g^{2}(T, \mu)$ is the two loop QCD running coupling constant at finite temperature $(T)$ and quark chemical potential $(\mu)[81,83]$,

$$
\begin{aligned}
g^{2}(T, \mu)= & \frac{24 \pi^{2}}{\left(33-2 n_{f}\right) \ln \left(\frac{T}{\Lambda_{T}} \sqrt{1+a \frac{\mu^{2}}{T^{2}}}\right)} \\
& \times\left(1-\frac{3\left(153-19 n_{f}\right)}{\left(33-2 n_{f}\right)^{2}} \frac{\ln \left(2 \ln \left(\frac{T}{\Lambda_{T}} \sqrt{1+a \frac{\mu^{2}}{T^{2}}}\right)\right)}{\ln \left(\frac{T}{\Lambda_{T}} \sqrt{1+a \frac{\mu^{2}}{T^{2}}}\right)}\right),
\end{aligned}
$$

where $\Lambda_{T}$ is the QCD scale parameter and $a=1 / \pi^{2}$.

The relaxation time $\tau$ of quarks (antiquarks) and gluon can be given by the following expression $[5,100]$ :

$$
\tau_{q(\bar{q})}=\frac{1}{5.1 T \alpha_{s}^{2} \ln \left(\frac{1}{\alpha_{s}}\right)\left(1+0.12\left(2 n_{f}+1\right)\right)}
$$

and

$$
\tau_{g}=\frac{1}{22.5 T \alpha_{s}^{2} \ln \left(\frac{1}{\alpha_{s}}\right)\left(1+0.06 n_{f}\right)},
$$

where $\alpha_{s}(T, \mu)=\frac{g^{2}(T, \mu)}{4 \pi}, g^{2}(T, \mu)$ is the temperature $(T)$, and quark chemical potential $(\mu)$ dependent strong coupling constant. It is important to note that the above mentioned relaxation time, as given in Eqs. (17) and (18), has been derived in massless case and for $\mu \ll T$. It has been argued in Ref. [118] that the effect of mass on the scattering cross sections is small. Thus, mass has nonqualitative difference in the relaxation time as well as on transport coefficient in 
TABLE I. Fitting parameters for $z_{q}$ and $z_{g}$ [122].

\begin{tabular}{lcccr}
\hline \hline$z_{g, q}$ & $a_{g, q}$ & $b_{g, q}$ & $a_{g, q}^{\prime}$ & $b_{g, q}^{\prime}$ \\
\hline Gluon & $0.803 \pm 0.009$ & $1.837 \pm 0.039$ & $0.978 \pm 0.007$ & $0.942 \pm 0.035$ \\
Quark & $0.810 \pm 0.010$ & $1.721 \pm 0.040$ & $0.960 \pm 0.007$ & $0.846 \pm 0.033$ \\
\hline \hline
\end{tabular}

the quasiparticle model. In this model, light quark masses have been chosen to be 0.1 times the strange quark mass, which is consistent with the chiral perturbation theory results [119-121]. The parameters $\Lambda_{T} / T_{c}$ and the strange quark mass can be adjusted to fit the lattice data [119]. The fitted parameters are $\Lambda_{T} / T_{c}=0.35$, with $T_{c}=200 \mathrm{MeV}$ and $m_{s 0}=80 \mathrm{MeV}[83]$.

\section{B. Quasiparticle model II (QPM II)}

In this model, the basic ansatz is that lattice QCD EOS can be reproduced in terms of noninteracting quasiparticle degrees of freedom having effective fugacities $\left(z_{q}, z_{g}\right)$ which encodes all the interaction effects of the particles in the system. These effective fugacities enter through the equilibrium distribution function of gluons and quarks (antiquarks) at finite temperature and vanishing baryon chemical potential, which can be expressed as [122]

$$
\begin{gathered}
f_{0}^{g}=\frac{z_{g} \exp (-\beta p)}{1-z_{g} \exp (-\beta p)}, \\
f_{0}^{q / \bar{q}}=\frac{z_{q} \exp (-\beta \epsilon(p))}{1+z_{q} \exp (-\beta \epsilon(p))}=\frac{z_{q} \exp \left(-\beta \sqrt{p^{2}+m^{2}}\right)}{1+z_{q} \exp \left(-\beta \sqrt{p^{2}+m^{2}}\right)} .
\end{gathered}
$$

From Eqs. (19) and (20), one gets the equilibrium distribution function of ideal quarks and gluon in the limit when quark/antiquark fugacity $\left(z_{q}\right)$ and gluons fugacity $\left(z_{g}\right)$ approach unity, i.e., $z_{q} \sim z_{g} \sim 1$.0. For the complete information of the quasiparticle distribution functions as given in Eqs. (19) and (20), one requires the temperature dependence of the fugacities $z_{q}$ and $z_{g}$. Temperature dependence of fugacities $z_{q}$ and $z_{g}$ can be obtained by matching the thermodynamic properties of this quasiparticle model with that of lattice QCD results. Fitting the thermodynamic properties of this model with lattice QCD data, for phenomenological purpose one can get a parametric form for $z_{q}$ and $z_{g}$ as a function of temperature [122]. Following Ref. [122], the temperature dependence of the fugacities $z_{q}$ and $z_{g}$ can be given as

$$
\begin{aligned}
& z_{q, g}=a_{q, g} \exp \left(-b_{q, g} / x^{5}\right), \quad \text { for, } x<x_{q, g}, \\
& z_{q, g}=a_{q, g}^{\prime} \exp \left(-b_{q, g}^{\prime} / x^{2}\right), \quad \text { for, } x>x_{q, g}, \\
& x_{q, g} \equiv T_{q, g} / T_{c} \sim 1.70,1.68,
\end{aligned}
$$

where the fitting parameters $a_{q, g}, a_{q, g}^{\prime}, b_{q, g}, b_{q, g}^{\prime}$ are given in Table I [122]. In this investigation, for simplicity, we have considered $T_{q, g} / T_{c}=T / T_{c} \sim 1.70$ and the central values of all the fitting parameters for $z_{q}$ and $z_{g}$. Variation of $z_{q}$ and $z_{g}$ with temperature as shown in the Fig. 1 is consistent with the estimate of $z_{q}$ and $z_{g}$, as given in Ref. [122].

It can be shown that effective fugacity parameters in the equilibrium distribution function affect single particle dispersion relation in the following way [122]:

$$
\begin{gathered}
\omega_{p}^{g}=p+T^{2} \partial_{T} \ln \left(z_{g}\right), \\
\omega_{p}^{q}=\sqrt{p^{2}+m^{2}}+T^{2} \partial_{T} \ln \left(z_{q}\right) .
\end{gathered}
$$

Although the dispersion relations are modified, it is important to note that in effective fugacity quasiparticle model, group velocity of the quasiparticles remains unchanged, i.e.,

$$
\vec{v}=\partial_{\vec{p}} \omega_{p}=\partial_{\vec{p}} \epsilon(p)=\frac{\vec{p}}{\epsilon(p)} .
$$

This model can be extended at finite quark chemical potential by introducing quark chemical potential in quark/ antiquark distribution function [123],

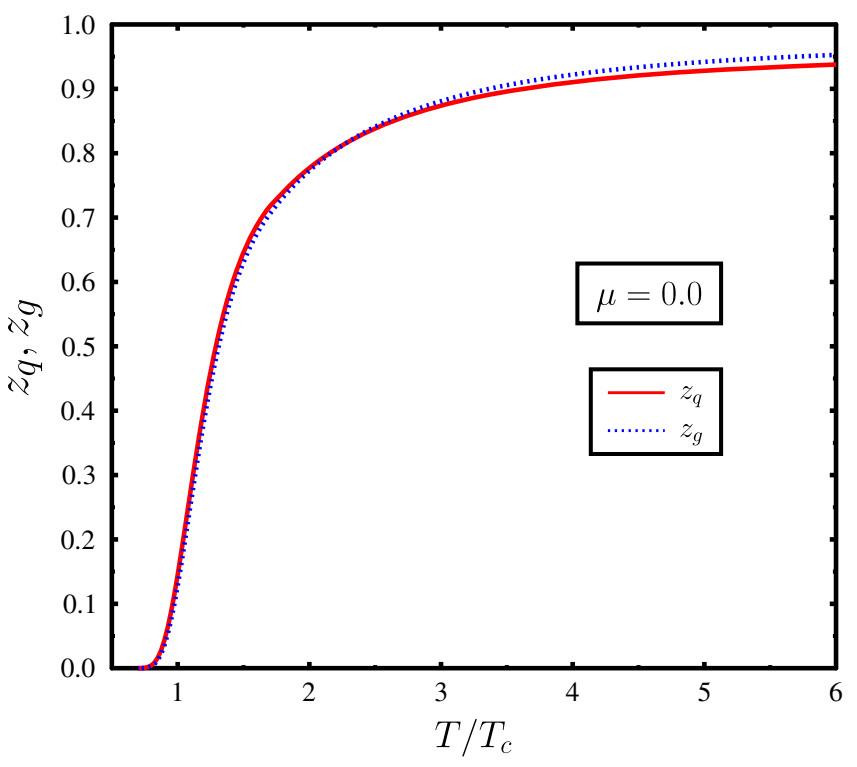

FIG. 1. Variation of $z_{q}$ and $z_{g}$ with temperature $(T)$. 


$$
f_{0}^{q / \bar{q}}=\frac{z_{q} \exp (-\beta(\epsilon(p) \mp \mu))}{1+z_{g} \exp (-\beta(\epsilon(p) \mp \mu))}=\frac{z_{q} \exp \left(-\beta\left(\sqrt{p^{2}+m^{2}} \mp \mu\right)\right)}{1+z_{q} \exp \left(-\beta\left(\sqrt{p^{2}+m^{2}} \mp \mu\right)\right)}
$$

It is important to note that although quark chemical potential is introduced in the distribution function at finite chemical potential, fugacities are assumed to be independent of chemical potential as they are fixed with lattice data at finite temperature and zero chemical potential.

The thermal averaged relaxation time of quarks, antiquarks, and gluons at finite temperature and chemical potential in the effective fugacity quasiparticle model has been considered as [123,124]

$$
\begin{aligned}
\tau_{g}^{-1}= & g_{g} \int \frac{d^{3} \vec{p}_{g}}{(2 \pi)^{3}} f_{0}^{g}\left(1+f_{0}^{g}\right)\left(\frac{9 g_{\mathrm{eff}}^{4}}{16 \pi\langle s\rangle_{g g}}\left[\ln \frac{\langle s\rangle_{g g}}{k^{2}}-1.267\right]\right)+g_{q} \int \frac{d^{3} \vec{p}_{q}}{(2 \pi)^{3}} f_{0}^{q}\left(1-f_{0}^{q}\right)\left(\frac{g_{\mathrm{eff}}^{4}}{4 \pi\langle s\rangle_{g q}}\left[\ln \frac{\langle s\rangle_{g q}}{k^{2}}-1.287\right]\right) \\
& +g_{\bar{q}} \int \frac{d^{3} \vec{p}_{\bar{q}}}{(2 \pi)^{3}} f_{0}^{\bar{q}}\left(1-f_{0}^{\bar{q}}\right)\left(\frac{g_{\mathrm{eff}}^{4}}{4 \pi\langle s\rangle_{g \bar{q}}}\left[\ln \frac{\langle s\rangle_{g \bar{q}}}{k^{2}}-1.287\right]\right), \\
\tau_{q}^{-1}= & g_{g} \int \frac{d^{3} \vec{p}_{g}}{(2 \pi)^{3}} f_{0}^{g}\left(1+f_{0}^{g}\right)\left(\frac{g_{\mathrm{eff}}^{4}}{4 \pi\langle s\rangle_{q g}}\left[\ln \frac{\langle s\rangle_{q g}}{k^{2}}-1.287\right]\right)+g_{q} \int \frac{d^{3} \vec{p}_{q}}{(2 \pi)^{3}} f_{0}^{q}\left(1-f_{0}^{q}\right)\left(\frac{g_{\mathrm{eff}}^{4}}{9 \pi\langle s\rangle_{q q}}\left[\ln \frac{\langle s\rangle_{q q}}{k^{2}}-1.417\right]\right) \\
& +g_{\bar{q}} \int \frac{d^{3} \vec{p}_{\bar{q}}}{(2 \pi)^{3}} f_{0}^{\bar{q}}\left(1-f_{0}^{\bar{q}}\right)\left(\frac{g_{\mathrm{eff}}^{4}}{9 \pi\langle s\rangle_{q \bar{q}}}\left[\ln \frac{\langle s\rangle_{q \bar{q}}}{k^{2}}-1.417\right]\right), \\
\tau_{\bar{q}}^{-1}= & g_{g} \int \frac{d^{3} \vec{p}_{g}}{(2 \pi)^{3}} f_{0}^{g}\left(1+f_{0}^{g}\right)\left(\frac{g_{\mathrm{eff}}^{4}}{4 \pi\langle s\rangle_{\bar{q} g}}\left[\ln \frac{\langle s\rangle_{\bar{q} g}}{k^{2}}-1.287\right]\right)+g_{q} \int \frac{d^{3} \vec{p}_{q}}{(2 \pi)^{3}} f_{0}^{q}\left(1-f_{0}^{q}\right)\left(\frac{g_{\mathrm{eff}}^{4}}{9 \pi\langle s\rangle_{\bar{q} q}}\left[\ln \frac{\langle s\rangle_{\bar{q} q}}{k^{2}}-1.417\right]\right) \\
& +g_{\bar{q}} \int \frac{d^{3} \vec{p}_{\bar{q}}}{(2 \pi)^{3}} f_{0}^{\bar{q}}\left(1-f_{0}^{\bar{q}}\right)\left(\frac{g_{\mathrm{eff}}^{4}}{9 \pi\langle s\rangle_{\bar{q} \bar{q}}}\left[\ln \frac{\langle s\rangle_{\bar{q} \bar{q}}}{k^{2}}-1.417\right]\right),
\end{aligned}
$$

where thermal average of the quantity $s$ is denoted as $\langle s\rangle_{k l}=2\left\langle p_{k}\right\rangle\left\langle p_{l}\right\rangle$, with $\left\langle p_{k}\right\rangle=\frac{\int \frac{d^{3} p_{k}}{(2 \pi)^{3}} \vec{p}_{k} \mid f_{0}^{k}}{\int \frac{d^{3} p_{k}}{(2 \pi)^{3}} f_{0}^{k}}$ and $k^{2}=g_{\mathrm{eff}}^{2} T^{2}$. Effective strong coupling constant $g_{\text {eff }}$ in this model can be determined using charge renormalization, by computing the Debye mass in the medium within the framework of effective fugacity quasiparticle model and comparing it to the hard thermal loop results. For $\mu / T \equiv \tilde{\mu}<1$, effective strong coupling constant can be shown to be [123]

$$
\alpha_{S_{\mathrm{eff}}}(T, \mu)=\frac{g_{\mathrm{eff}}^{2}}{4 \pi}=\alpha_{s}(T, \mu) \frac{\frac{2 N_{c}}{\pi^{2}} \operatorname{PolyLog}\left[2, z_{g}\right]-\frac{2 N_{f}}{\pi^{2}} \operatorname{PolyLog}\left[2,-z_{q}\right]+\tilde{\mu}^{2}\left(\frac{N_{f}}{\pi^{2}} \frac{z_{q}}{1+z_{q}}\right)}{\left(\frac{N_{c}}{3}+\frac{N_{f}}{6}\right)+\tilde{\mu}^{2} \frac{N_{f}}{2 \pi^{2}}},
$$

where $\alpha_{s}(T, \mu)$ is the temperature and chemical potentialdependent strong coupling constant [83]. In this investigation, we have considered strange quark mass $\left(m_{s}\right)$ to be $80 \mathrm{MeV}$ and the light quark masses are taken as one tenth of the strange quark mass for QPM II. QCD transition temperature is taken as $T_{c}=200 \mathrm{MeV}$ [119].

\section{RESULTS AND DISCUSSIONS}

In Fig. 2, we show the variation of the normalized electrical conductivity $\left(\sigma^{\mathrm{el}} / T\right)$ with temperature at vanishing magnetic field $(e B=0.0)$ and quark chemical potential $(\mu)$ for QPM I and QPM II. From this figure, it is clear that normalized electrical conductivity $\left(\sigma^{\mathrm{el}} / T\right)$ in QPM I is consistent with the lattice QCD data given by Amato et al.
[26]. On the other hand, normalized electrical conductivity $\left(\sigma^{\mathrm{el}} / T\right)$ as estimated in QPM II is consistent with the lattice QCD data given by Gupta et al. [27]. For comparison, we have also shown in Fig. 2 normalized electrical conductivity obtained in Nambu-Jona-Lassinio model as given by Marty et al. [28]. Values of the normalized electrical conductivity in these two quasiparticle models are order of magnitude different. This is because electrical conductivity is proportional to the relaxation time, which is order of magnitude larger in QPM II with respect to QPM I as can be seen from Fig. 3 .

In Figs. 3(a) and 3(b), we show the variation of relaxation time of $u$ quarks with temperature $(T)$ and quark chemical potential $(\mu)$. For the QPM I, relaxation time of the quarks and gluons, as given in Eqs. (17) and (18), was 


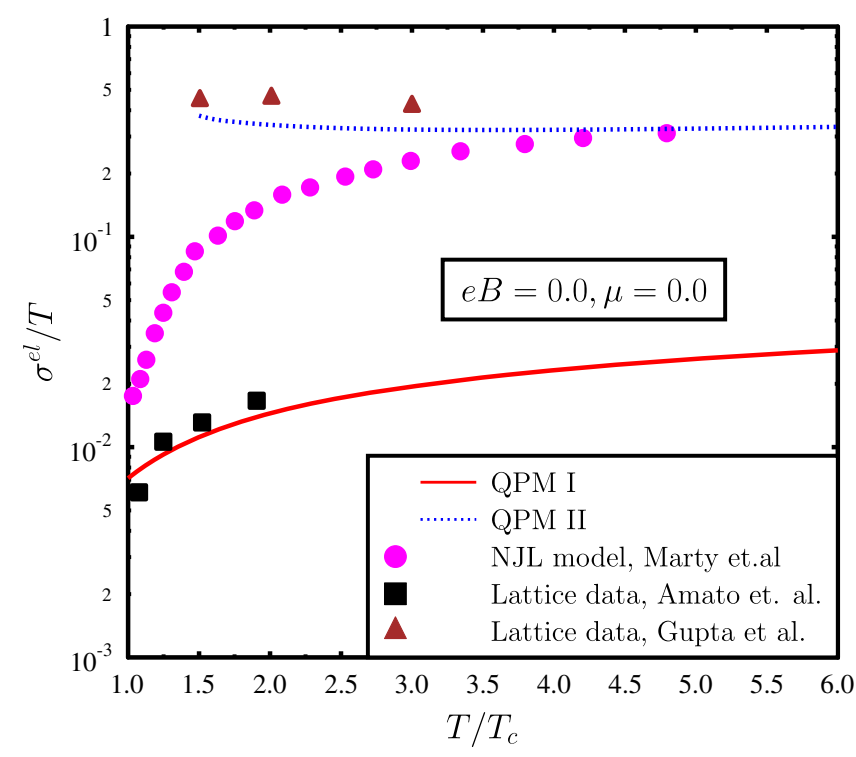

FIG. 2. Variation of normalized electrical conductivity $\left(\sigma^{\mathrm{el}} / T\right)$ with temperature $(T)$ at vanishing magnetic field and vanishing quark chemical potential. Lattice data are also shown in this plot for comparison. Value of $\sigma^{\mathrm{el}} / T$ in QPM I is consistent with lattice data given by Amato et al. [26]. On the other hand, $\sigma^{\mathrm{el}} / T$ as estimated in QPM II is consistent with the lattice QCD data given by Gupta et al. [27].

derived for the case of $\mu \ll T$ [5]. Hence, throughout this investigation, we have considered relaxation time in QPM I for vanishing quark chemical potential. It is important to mention that in this investigation we have not considered the effect of magnetic field on the relaxation time as magnetic field is not the dominant scale. From Fig. 3, it is clear that the thermal average relaxation time in QPM II

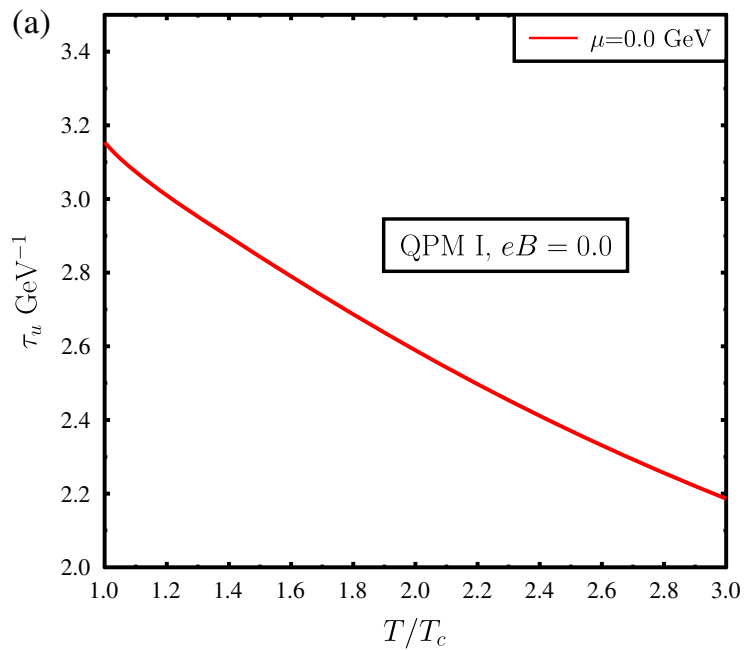

is order of magnitude larger that its counterpart in QPM I. This is because of the different quasiparticle nature of the partons in these models, as can be seen from Eqs. (17) and (27). This apart from Figs. 3(a) and 3(b) we can also see that with increasing temperature relaxation time decreases. Physically, this means with increasing temperature as the number density of the partons increases collision rate increases. From Fig. 3(b), it is clear that with increasing chemical potential relaxation time decreases in QPM II. Although the dependence of relaxation time on the chemical potential in QPM II is convoluted as can be seen from Eq. (27), but physically one can understand the variation of relaxation time with quark chemical potential in the following way. With increasing chemical potential, number density of the scatterer increases due to increasing number density of the particles. Hence, interaction rate increases with increasing quark chemical potential, which gives rise to decreasing behavior of relaxation time with increasing quark chemical potential.

In Fig. 4, we show the variation of normalized electrical conductivity with temperature at vanishing quark chemical potential but with finite magnetic field. From Figs. 4(a) and 4(b), we see that with increasing magnetic field normalized electrical conductivity $\left(\sigma^{\mathrm{el}} / T\right)$ decreases. In QPM II, the decrease in $\sigma^{\mathrm{el}} / T$ is much larger than its counterpart in QPM I. This behavior can be understood from Eq. (8). Since in QPM I value of the relaxation time is order of magnitude smaller than the relaxation time in QPM II, $\omega_{c} \tau$ in the denominator in Eq. (8) is larger in QPM II. This gives rise to larger decrease in the normalized electrical conductivity in QPM II with respect to QPM I. Physically, electrical conductivity decreases with magnetic field because with increasing magnetic field more particles

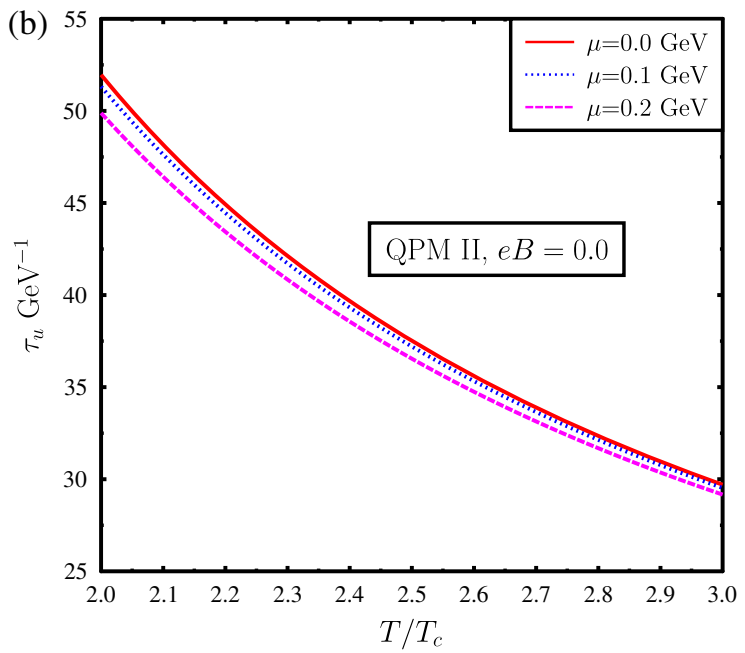

FIG. 3. Plot (a): variation of thermal averaged relaxation time of $u$ quark with temperature for vanishing magnetic field and quark chemical potential in QPM I. Plot (b): variation of relaxation time of $u$ quark with temperature for vanishing magnetic field but with different values quark chemical potential in QPM II. From plot (a) and plot (b), we see that with increasing temperature thermalized relaxation time decreases for both the quasiparticle models. For QPM II, relaxation time decreases with increase in quark chemical potential. 

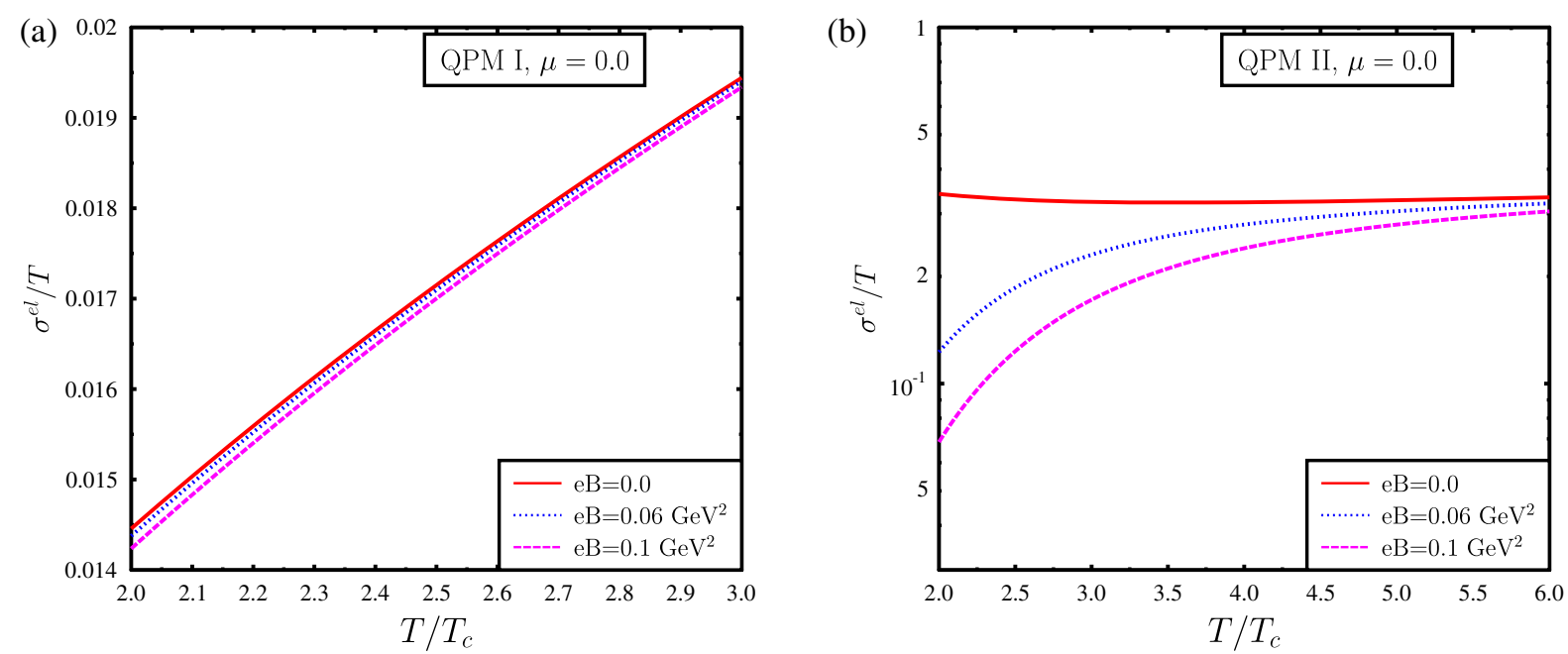

FIG. 4. Plot (a): variation of normalized electrical conductivity $\left(\sigma^{\mathrm{el}} / T\right)$ with temperature for vanishing quark chemical potential but with different values of magnetic fields in QPM I. Plot (b): variation of normalized electrical conductivity $\left(\sigma^{\mathrm{el}} / T\right)$ with temperature for vanishing quark chemical potential but with different values of magnetic fields in QPM II. From plot (a) and plot (b), we can see that for both the quasiparticle models, normalized electrical conductivity decreases with increasing magnetic field. Decrease of normalized electrical conductivity is significantly larger in QPM II with respect to its counterpart in QPM I.

are deviated from the direction of electric field, hence reduction in electrical current. Variation of normalized electric conductivity with temperature for a fixed magnetic field and quark chemical potential depends crucially on the temperature dependence of relaxation time and the equilibrium distribution function. For the range of quark chemical potential, temperature, and magnetic field considered in this investigation, from Fig. 4(a) we can see that with temperature $\sigma^{\mathrm{el}} / T$ increases. This increasing behavior of $\sigma^{\mathrm{el}} / T$ is predominately due to the Boltzmann factor $\exp (-\epsilon(p) / T)$ in the distribution function, which increases with increasing temperature. Similarly, for QPM II, with

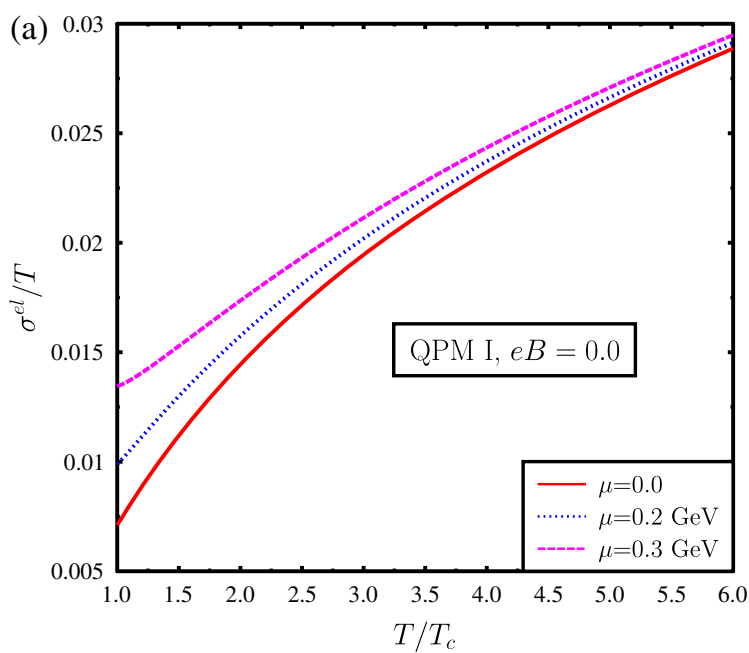

temperature normalized, electrical conductivity increases for nonvanishing magnetic field as can be seen in Fig. 4(b). With increasing temperature, the relaxation time decreases and the Boltzmann factor in the distribution function increases, giving rise to this increasing behavior of normalized electrical conductivity at nonvanishing magnetic field in QPM II.

Next, we show the variation of normalized electrical conductivity with temperature for vanishing magnetic field but with finite quark chemical potential in Fig. 5. From Figs. 5(a) and 5(b), we can see that for both the quasiparticle models $\sigma^{\mathrm{el}} / T$ increases with quark chemical potential.

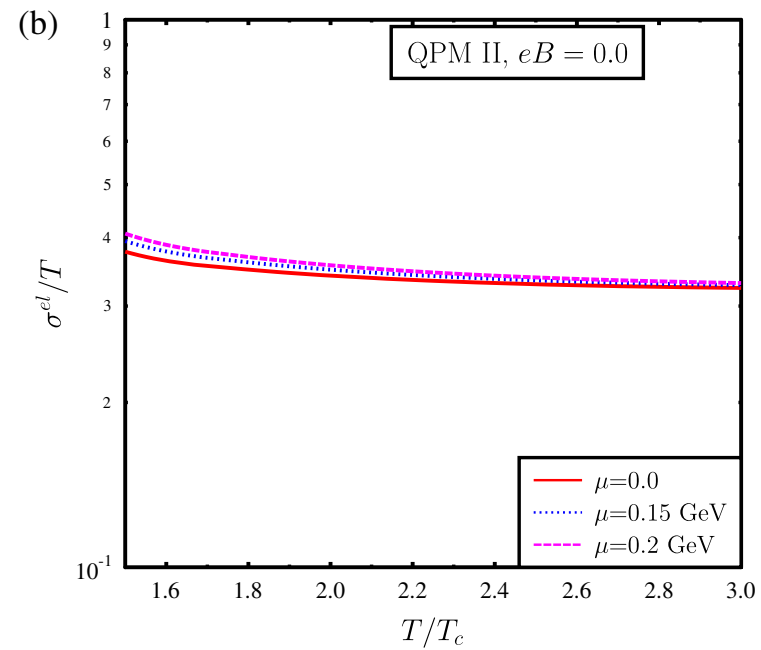

FIG. 5. Plot (a): variation of normalized electrical conductivity $\left(\sigma^{\mathrm{el}} / T\right)$ with temperature for vanishing magnetic field and different values of quark chemical potential in QPM I. Plot (b): variation of normalized electrical conductivity $\left(\sigma^{\text {el }} / T\right)$ with temperature for vanishing magnetic field and different values of quark chemical potential in QPM II. For QPM I [plot (a)] and QPM II [plot (b)], with increasing quark chemical normalized electrical conductivity increases. 

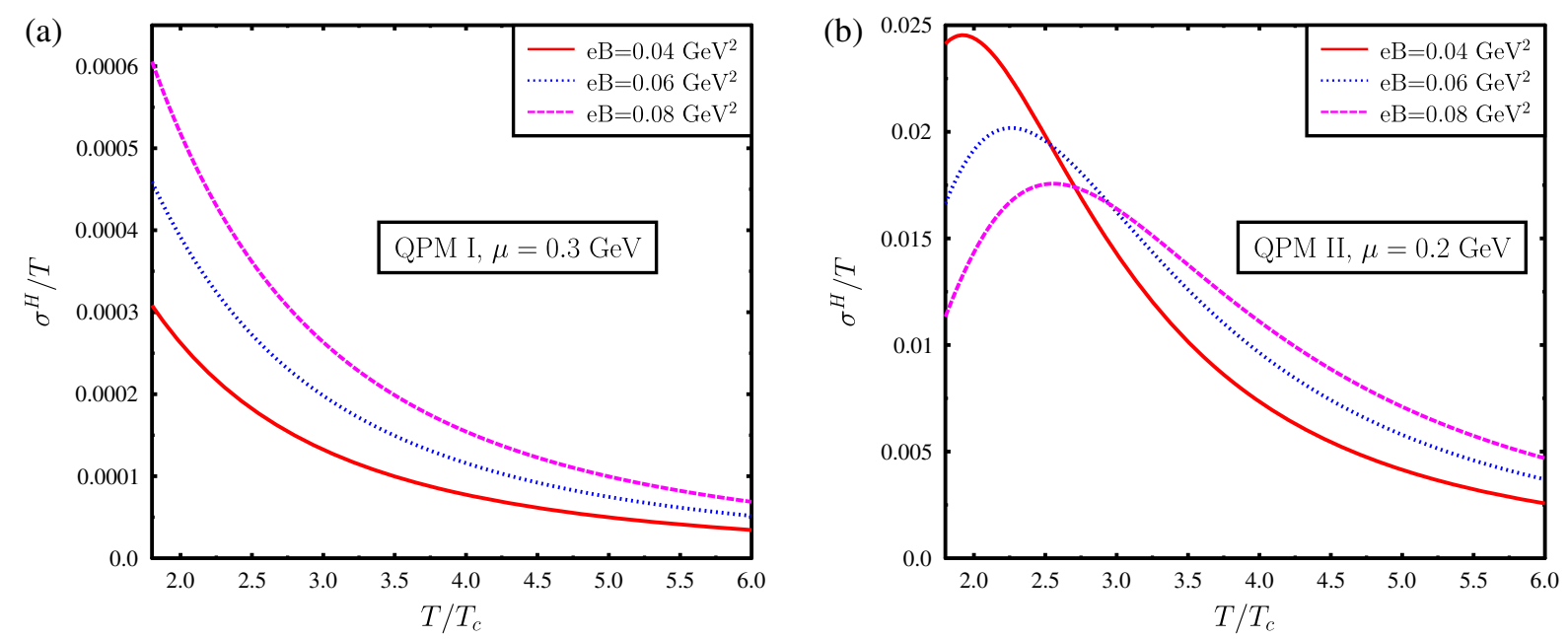

FIG. 6. Plot (a): variation of normalized Hall conductivity $\left(\sigma^{H} / T\right)$ with temperature for a nonvanishing quark chemical potential and various values of magnetic field in QPM I. Plot (b): variation of normalized Hall conductivity $\left(\sigma^{H} / T\right)$ with temperature for a nonvanishing quark chemical potential and various values of magnetic field in QPM II. For QPM I, as shown in plot (a), $\sigma^{H} / T$ increases with magnetic field. Contrary to QPM I, in QPM II, as shown in plot (b) for relatively low temperature, normalized Hall conductivity decreases with magnetic field and for higher temperature it increases with magnetic field. For a fixed value of magnetic field and quark chemical potential in QPM I [plot(a)], $\sigma^{H} / T$ decreases with temperature. On the other hand, for QPM II [plot (b)], $\sigma^{H} / T$ first increases with temperature for relatively small temperature and eventually it decreases with temperature at large temperature.

Variation of normalized electrical conductivity with quark chemical potential intimately connected with the variation of relaxation time with quark chemical potential and the Boltzmann factor $\exp ( \pm \mu / T)$ in the equilibrium distribution function. For QPM I, thermal average relaxation time has been calculated for vanishing quark chemical potential. Hence, relaxation time does not change with quark chemical potential. At finite chemical potential, number density of quarks is larger than antiquarks; hence, in the total electrical conductivity, contribution from the quarks is larger with respect to the antiquarks. With increasing quark chemical potential, Boltzmann factor in the distribution function increases. This increasing behavior of the Boltzmann factor in the distribution function results in the increasing behavior of normalized electrical conductivity with quark chemical potential in QPM I. On the other hand, for QPM II, from Fig. 3(b), we can see that with increasing quark chemical potential relaxation time decreases. But this decrease in the relaxation time with increasing quark chemical potential is compensated by the Boltzmann factor $[\exp (\mu / T)]$ in the distribution function. Hence, in QPM II, normalized electrical conductivity increases with quark chemical potential. It is also important to note that at relatively high temperature normalized electrical conductivity does not change by a large amount with chemical potential. This is because at high temperature Boltzmann factor $[(\exp (\mu / T)]$ is not large within the temperature and chemical potential range considered here.

Now, we turn our focus to the variation of normalized Hall conductivity $\sigma^{H} / T$ with temperature. For vanishing quark chemical potential, the relaxation time of quarks and antiquarks is same. Hence, in the Hall conductivity, particles and antiparticle contributions are exact but opposite. So, the net Hall current at vanishing quark chemical potential is zero as can be explicitly seen in Eq. (9). Only for nonvanishing quark chemical potential, Hall conductivity has nonzero value. In Fig. 6, we show the variation of normalized Hall conductivity $\left(\sigma^{H} / T\right)$ with temperature for nonvanishing quark chemical potential and magnetic field. From Fig. 6(a), for QPM I, we see that for finite quark chemical potential Hall conductivity increases with magnetic field. However, for QPM II, Hall conductivity has a nonmonotonic behavior with temperature, as can be seen in Fig. 6(b), where at small temperature Hall conductivity decreases with increase in magnetic field and at relatively high temperature Hall conductivity increases with increase in magnetic field. This different behavior of Hall conductivity in QPM I and QPM II is mainly due to different values of relaxation time in these quasiparticle models. This behavior of $\sigma^{H} / T$ as shown in Fig. 6(b) with magnetic field is plausibility due to the factor $\frac{\omega_{c} \tau}{1+\left(\omega_{c} \tau\right)^{2}}$ in the Hall conductivity as can be seen from Eq. (9). At relatively small temperature, relaxation time is large and $\sigma^{H} / T \sim \frac{1}{\omega_{c}}$. On the other hand, at high temperature, relaxation time is smaller and $\sigma^{H} / T \sim \omega_{c}$. Thus, at smaller temperature with increasing magnetic field, normalized Hall conductivity decreases and at high temperature it increases with magnetic field in QPM II. As we have already mentioned in QPM I, relaxation time is order of magnitude smaller than that of in QPM II; hence, in this case, $\sigma^{H} / T \sim \omega_{c}$ for the range of temperature, chemical potential, and magnetic field considered here. Hence, in QPM I, Hall conductivity 

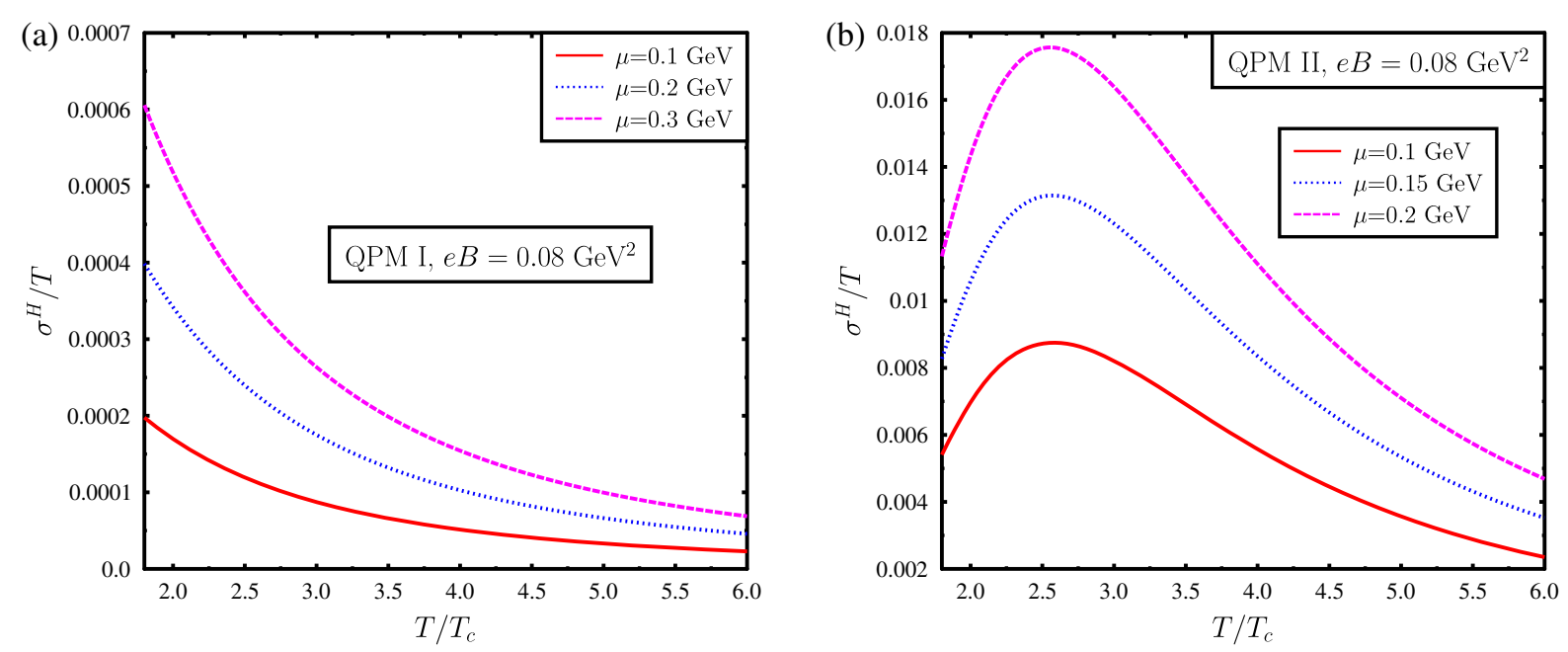

FIG. 7. Plot (a): variation of normalized Hall conductivity $\left(\sigma^{H} / T\right)$ with temperature $(T)$ for various values of quark chemical potential $(\mu)$ and fixed nonvanishing magnetic field in QPM I. Plot (b): variation of normalized Hall conductivity $\left(\sigma^{H} / T\right)$ with temperature $(T)$ for various values of quark chemical potential $(\mu)$ and fixed nonvanishing magnetic field in QPM II. From these plots, we can see that with increasing quark chemical potential $\sigma^{H} / T$ increases. For QPM I, with increasing temperature, normalized Hall conductivity decreases. On the other hand, for QPM II, normalized Hall conductivity varies nonmonotonically with temperature.

increases with magnetic field. For a fixed magnetic field, variation of normalized Hall conductivity with temperature is rather convoluted. In QPM II, for a fixed magnetic field, at relatively low temperature due to large relaxation time, $\sigma^{H} / T \sim f_{0}\left(1-f_{0}\right) / T^{2}$, which increases with increasing temperature for the parameter range considered in this work. On the other hand, at relatively high temperature, due to small relaxation time, $\sigma^{H} / T \sim \tau^{2} f_{0}\left(1-f_{0}\right) / T^{2}$, which decreases with increasing temperature.

In Fig. 7, we show the variation of normalized hall conductivity $\left(\sigma^{H} / T\right)$ with temperature for different values of quark chemical potential and finite magnetic field. For Figs. 7(a) and 7(b), it is clear that with increasing quark chemical potential Hall conductivity increases. At vanishing quark chemical potential, Hall conductivity is zero because of the exact cancellation of Hall current of particles and its antiparticles. At finite quark chemical potential, number density of particles is larger than that of antiparticles. Hence, at finite quark chemical potential, there is a net Hall current and nonvanishing Hall conductivity. With increasing quark chemical potential, contribution of the particles increases, which results in the increasing behavior of $\sigma^{H} / T$ with quark chemical potential, as can be seen in Fig. 7. Similar to Fig. 6 for a fixed chemical potential and magnetic field, variation of normalized Hall conductivity with temperature is convoluted and it depends upon various factors, e.g., relaxation time, distribution function, etc.

\section{CONCLUSIONS}

In this investigation, we study the electrical conductivity and Hall conductivity in the presence of magnetic field for quark gluon plasma within the framework of quasiparticle models. Here we have considered two specific quasiparticle models of QGP, QPM I, where the quasiparticle nature of the quarks and gluon are encoded in thermal mass of the quasi partons, and QPM II, where the quasiparticle nature is encoded in the effective fugacity parameter in the distribution function. Both of these models have been explored earlier in literature. We found that with increasing magnetic field normalized electrical conductivity $\left(\sigma^{\mathrm{el}} / T\right)$ decreases in both quasiparticle models. However, due to widely different values of relaxation time in these models, decrease in normalized electrical conductivity with magnetic field is larger in QPM II than its counterpart in QPM I. In both these models, thermal averaged relaxation time decreases with temperature. In QPM II, relaxation time decreases with increasing quark chemical potential, plausibility due to increasing number density of quasi partons and increasing interaction rates. In both models, normalized electrical conductivity increases with increasing quark chemical potential. For a pair plasma at vanishing quark chemical potential, Hall current due to particles and antiparticles cancels each other. Hence, Hall conductivity can have a nonzero value only for finite quark chemical potential. In QPM I, with increasing magnetic field, normalized Hall conductivity $\left(\sigma^{H} / T\right)$ increases. On the other hand, for QPM II, at relatively small temperature, $\sigma^{H} / T$ decreases with increasing magnetic field and at high temperature $\sigma^{H} / T$ increases with increase in magnetic field. Finally, with finite magnetic field, $\sigma^{H} / T$ increases with increase in quark chemical potential. This is due to the fact that at finite quark chemical potential contributions of the particles are larger than their antiparticles in the net Hall conductivity and with increasing chemical potential 
contribution of the quarks increases, resulting in an increasing behavior of $\sigma^{H} / T$ with quark chemical potential. Our results clearly demonstrate that while the two quasiparticle models considered here are consistent with lattice results regarding thermodynamics, the resulting transport coefficients are quite different from the numerical values for the same differing by an order of magnitude.

\section{ACKNOWLEDGMENTS}

R. K. M. would like to thank Theoretical Physics Division of Physical Research Laboratory, Ahmedabad for support and local hospitality for her visit, during which this problem was initiated. Also, R. K. M. would like to thank Basanta K. Nandi and Sadhana Dash for constant support and encouragement.
[1] U. W. Heinz and R. Snellings, Annu. Rev. Nucl. Part. Sci. 63, 123 (2013).

[2] P. Romatschke and U. Romatschke, Phys. Rev. Lett. 99, 172301 (2007).

[3] P. K. Kovtun, D. T. Son, and A. O. Starinets, Phys. Rev. Lett. 94, 111601 (2005).

[4] S. Gavin, Nucl. Phys. A435, 826 (1985).

[5] A. Hosoya and K. Kajantie, Nucl. Phys. B250, 666 (1985).

[6] A. Dobado and J. M. Torres-Rincon, Phys. Rev. D 86, 074021 (2012).

[7] C. Sasaki and K. Redlich, Phys. Rev. C 79, 055207 (2009).

[8] C. Sasaki and K. Redlich, Nucl. Phys. A832, 62 (2010).

[9] F. Karsch, D. Kharzeev, and K. Tuchin, Phys. Lett. B 663, 217 (2008).

[10] S. I. Finazzo, R. Rougemont, H. Marrochio, and J. Noronha, J. High Energy Phys. 02 (2015) 051.

[11] A. Wiranata and M. Prakash, Nucl. Phys. A830, 219C (2009).

[12] S. Jeon and L. Yaffe, Phys. Rev. D 53, 5799 (1996).

[13] D. E. Kharzeev, L. D. McLerran, and H. J. Warringa, Nucl. Phys. A803, 227 (2008).

[14] V. Skokov, A. Yu. Illarionov, and V. Toneev, Int. J. Mod. Phys. A 24, 5925 (2009).

[15] G. Inghirami, L. Del Zanna, A. Beraudo, M. H. Moghaddam, F. Becattini, and M. Bleicher, Eur. Phys. J. C 76, 659 (2016).

[16] A. Das, S. S. Dave, P. S. Saumia, and A. M. Srivastava, Phys. Rev. C 96, 034902 (2017).

[17] K. Tuchin, Phys. Rev. C 83, 017901 (2011); 82, 034904 (2010).

[18] M. Greif, C. Greiner, and G. S. Denicol, Phys. Rev. D 93, 096012 (2016).

[19] M. Greif, I. Bouras, C. Greiner, and Z. Xu, Phys. Rev. D 90, 094014 (2014).

[20] A. Puglisi, S. Plumari, and V. Greco, Phys. Lett. B 751, 326 (2015).

[21] A. Puglisi, S. Plumari, and V. Greco, Phys. Rev. D 90, 114009 (2014).

[22] W. Cassing, O. Linnyk, T. Steinert, and V. Ozvenchuk, Phys. Rev. Lett. 110, 182301 (2013).

[23] T. Steinert and W. Cassing, Phys. Rev. C 89, 035203 (2014).

[24] G. Aarts, C. Allton, A. Amato, P. Giudice, S. Hands, and J.-I. Skullerud, J. High Energy Phys. 02 (2015) 186.
[25] G. Aarts, C. Allton, J. Foley, S. Hands, and S. Kim, Phys. Rev. Lett. 99, 022002 (2007).

[26] A. Amato, G. Aarts, C. Allton, P. Giudice, S. Hands, and J.-I. Skullerud, Phys. Rev. Lett. 111, 172001 (2013).

[27] S. Gupta, Phys. Lett. B 597, 57 (2004).

[28] R. Marty, E. Bratkovskaya, W. Cassing, J. Aichelin, and H. Berrehrah, Phys. Rev. C 88, 045204 (2013).

[29] Y. Burnier and M. Laine, Eur. Phys. J. C 72, 1902 (2012).

[30] H.-T. Ding, A. Francis, O. Kaczmarek, F. Karsch, E. Laermann, and W. Soeldner, Phys. Rev. D 83, 034504 (2011).

[31] O. Kaczmarek and M. Muller, Proc. Sci., LATTICE2013 (2014) 175.

[32] S.-X. Qin, Phys. Lett. B 742, 358 (2015).

[33] D. Fern andez-Fraile and A. G. Nicola, Phys. Rev. D 73, 045025 (2006).

[34] Strongly Interacting Matter in Magnetic Field, edited by D. Kharzeev, K. Landsteiner, A. Schmitt, and H. Yee, Lecture Notes in Physics Vol. 871 (Springer-Verlag, Berlin, Heidelberg, 2013).

[35] M. Greif, J. A. Fotakis, G. S. Denicol, and C. Greiner, Phys. Rev. Lett. 120, 242301 (2018).

[36] M. Prakash, M. Prakash, R. Venugopalan, and G. Welke, Phys. Rep. 227, 321 (1993).

[37] A. Wiranata and M. Prakash, Phys. Rev. C 85, 054908 (2012).

[38] P. Chakraborty and J. I. Kapusta, Phys. Rev. C 83, 014906 (2011).

[39] A. S. Khvorostukhin, V. D. Toneev, and D. N. Voskresensky, Nucl. Phys. A845, 106 (2010).

[40] S.Plumari, A. Paglisi, F. Scardina, and V. Greco, Phys. Rev. C 86, 054902 (2012).

[41] M. I. Gorenstein, M. Hauer, and O. N. Moroz, Phys. Rev. C 77, 024911 (2008).

[42] J. Noronha-Hostler, J. Noronha, and C. Greiner, Phys. Rev. C 86, 024913 (2012).

[43] S. K. Tiwari, P. K. Srivastava, and C. P. Singh, Phys. Rev. C 85, 014908 (2012).

[44] S. Ghosh, A. Lahiri, S. Majumder, R. Ray, and S. K. Ghosh, Phys. Rev. C 88, 068201 (2013).

[45] R. Lang, N. Kaiser, and W. Weise, Eur. Phys. J. A 51, 127 (2015).

[46] S. Ghosh, G. Krein, and S. Sarkar, Phys. Rev. C 89, 045201 (2014). 
[47] A. Wiranata, V. Koch, M. Prakash, and X. N. Wang, J. Phys. Conf. Ser. 509, 012049 (2014).

[48] A. Wiranata, M. Prakash, and P. Chakraborty, Central Eur. J. Phys. 10, 1349 (2012).

[49] A. Tawfik and M. Wahba, Ann. Phys. (Amsterdam) 522, 849 (2010).

[50] J. Noronha-Hostler, J. Noronha, and C. Greiner, Phys. Rev. Lett. 103, 172302 (2009).

[51] G. Kadam and H. Mishra, Nucl. Phys. A934, 133 (2015).

[52] G. Kadam, Mod. Phys. Lett. A 30, 1550031 (2015).

[53] S. Ghosh, Int. J. Mod. Phys. A 29, 1450054 (2014).

[54] N. Demir and A. Wiranata, J. Phys. Conf. Ser. 535, 012018 (2014).

[55] S. Ghosh, Phys. Rev. C 90, 025202 (2014).

[56] J.-B. Rose, J. M. Torres-Rincon, A. Schfer, D. R. Oliinychenko, and H. Petersen, Phys. Rev. C 97, 055204 (2018).

[57] C. Wesp, A. El, F. Reining, Z. Xu, I. Bouras, and C. Greiner, Phys. Rev. C 84, 054911 (2011).

[58] S. A. Bass et al., Prog. Part. Nucl. Phys. 41, 255 (1998).

[59] G. Kadam and H. Mishra, Phys. Rev. C 92, 035203 (2015).

[60] R. K. Mohapatra, H. Mishra, S. Dash, and B. K. Nandi, arXiv:1901.07238.

[61] P. Singha, A. Abhishek, G. Kadam, S. Ghosh, and H. Mishra, J. Phys. G 46, 015201 (2019).

[62] A. Abhishek, H. Mishra, and S. Ghosh, Phys. Rev. D 97, 014005 (2018).

[63] J. R. Bhatt, A. Das, and H. Mishra, Phys. Rev. D 99, 014015 (2019).

[64] G. S. Denicol, H. Niemi, I. Bouras, E. Molnar, Z. Xu, D. H. Rischke, and C. Greiner, Phys. Rev. D 89, 074005 (2014).

[65] J. I. Kapusta and J. M. Torres-Rincon, Phys. Rev. C 86, 054911 (2012).

[66] K. Tuchin, Phys. Rev. C 88, 024911 (2013).

[67] K. Tuchin, Adv. High Energy Phys. 2013, 490495 (2013).

[68] B. Feng, Phys. Rev. D 96, 036009 (2017).

[69] A. Das, H. Mishra, and R. K. Mohapatra, Phys. Rev. D 99, 094031 (2019).

[70] M. Bluhm, B. Kampfer, R. Schulze, and D. Seipt, Eur. Phys. J. C 49, 205 (2007).

[71] M. Bluhm, B. Kampfer, and K. Redlich, Phys. Lett. B 709, 77 (2012).

[72] M. Bluhm, B. Kampfer, and K. Redlich, Phys. Rev. C 84, 025201 (2011).

[73] M. Bluhm, B. Kampfer, and K. Redlich, Nucl. Phys. A830, 737c (2009).

[74] V. Mykhaylova, M. Bluhm, K. Redlich, and C. Sasaki, Phys. Rev. D 100, 034002 (2019).

[75] S. Plumari, W. M. Alberico, V. Greco, and C. Ratti, Phys. Rev. D 84, 094004 (2011).

[76] M. Ruggieri, P. Alba, P. Castorina, S. Plumari, C. Ratti, and V. Greco, Phys. Rev. D 86, 054007 (2012).

[77] L. Oliva, P. Castorina, V. Greco, and M. Ruggieri, Phys. Rev. D 88, 097502 (2013).

[78] H. Berrehrah, E. Bratkovskaya, T. Steinert, and W. Cassing, Int. J. Mod. Phys. E 25, 1642003 (2016).

[79] V. Goloviznin and H. Satz, Z. Phys. C 57, 671 (1993).

[80] M. I. Gorenstein and S. N. Yang, Phys. Rev. D 52, 5206 (1995).

[81] V. M. Bannur, Phys. Lett. B 647, 271 (2007).
[82] V. M. Bannur, Phys. Rev. C 75, 044905 (2007).

[83] L. L. Zhu and C. B. Yang, Nucl. Phys. A831, 49 (2009).

[84] E. P. Politis, C. E. Tsagkarakis, F. K. Diakonos, X. N. Maintas, and A. Tsapalis, Phys. Lett. B 763, 139 (2016).

[85] R. D. Pisarski, K. Kashiwa, and V. Skokov, Nucl. Phys. A904-905, 973c (2013).

[86] J. Cao, A.-M. Zhao, W.-M. Sun, and H.-S. Zong, Chin. Phys. Lett. 29, 061201 (2012).

[87] A.-M. Zhao, J. Cao, L.-J. Luo, W.-M. Sun, and H.-S. Zong, Mod. Phys. Lett. A 25, 47 (2010).

[88] V. Chandra and V. Ravishankar, Eur. Phys. J. C 64, 63 (2009).

[89] S. K. Ghosh, T. K. Mukherjee, and S. Raha, Mod. Phys. Lett. A 21, 2067 (2006).

[90] A. Peshier, B. Kampfer, O. P. Pavlenko, and G. Soff, Phys. Rev. D 54, 2399 (1996).

[91] C. Hamaguchi, Basic Semiconductor Physics (Springer, Berlin, Heidelberg, 2010).

[92] A. Kandus and C. G. Tsagas, Mon. Not. R. Astron. Soc. 385, 883 (2008).

[93] E. G. Blackman and G. B. Field, Phys. Rev. Lett. 71, 3481 (1993).

[94] N. Bessho and A. Bhattacharjee, Phys. Plasmas 14, 056503 (2007).

[95] See https://www.gsi.de/en/researchaccelerators/fair.htm.

[96] See http://nica.jinr.ru.

[97] V. Ozvenchuk, O. Linnyk, M. I. Gorenstein, E. L. Bratkovskaya, and W. Cassing, Phys. Rev. C 87, 064903 (2013).

[98] A. Puglisi, S. Plumari, and V. Greco, Phys. Lett. B 751, 326 (2015).

[99] P. K. Srivastava, L. Thakur, and B. K. Patra, Phys. Rev. C 91, 044903 (2015).

[100] L. Thakur, P. K. Srivastava, G. P. Kadam, M. George, and H. Mishra, Phys. Rev. D 95, 096009 (2017).

[101] S. Mitra and V. Chandra, Phys. Rev. D 97, 034032 (2018).

[102] V. Chandra, Phys. Rev. D 86, 114008 (2012).

[103] M. Kurian and V. Chandra, Phys. Rev. D 99, 116018 (2019).

[104] M. Kurian, S. Mitra, S. Ghosh, and V. Chandra, Eur. Phys. J. C 79, 134 (2019).

[105] M. Kurian and V. Chandra, Phys. Rev. D 97, 116008 (2018).

[106] L. Thakur and P. K. Srivastava, Phys. Rev. D 100, 076016 (2019).

[107] J. Dey, S. Satapathy, P. Murmu, and S. Ghosh, arXiv:1907 .11164 .

[108] J. Dey, S. Satapathy, A. Mishra, S. Paul, and S. Ghosh, arXiv:1908.04335.

[109] S. Satapathy, S. Paul, A. Anand, R. Kumar, and S. Ghosh, arXiv:1908.04330.

[110] B. Chatterjee, R. Rath, G. Sarwar, and R. Sahoo, arXiv: 1908.01121.

[111] K. Hattori, X.-G. Huang, D. H. Rischke, and D. Satow, Phys. Rev. D 96, 094009 (2017).

[112] K. Hattori, S. Li, D. Satow, and H.-U. Yee, Phys. Rev. D 95, 076008 (2017).

[113] G. Kadam, H. Mishra, and L. Thakur, Phys. Rev. D 98, 114001 (2018).

[114] A. Harutyunyan and A. Sedrakian, Phys. Rev. C 94, 025805 (2016). 
[115] G. S. Denicol, X.-G. Huang, E. Molnr, G. M. Monteiro, H. Niemi, J. Noronha, D. H. Rischke, and Q. Wang, Phys. Rev. D 98, 076009 (2018).

[116] G. S. Denicol, E. Molnr, H. Niemi, and D. H. Rischke, Phys. Rev. D 99, 056017 (2019).

[117] A. Peshier, B. Kampfer, and G. Scoff, Phys. Rev. C 61, 045203 (2000); Phys. Rev. D 66, 094003 (2002).

[118] H. Berrehrah, E. Bratkovskaya, W. Cassing, P. B. Gossiaux, J. Aichelin, and M. Bleicher, Phys. Rev. C 89, 054901 (2014).
[119] M. Cheng et al. Phys. Rev. D 77, 014511 (2008).

[120] C. Schmidt et al., Nucl. Phys. A820, 41C (2009).

[121] C. Miao and C. Schmidt, Proc. Sci., LATTICE2007 (2007) 175.

[122] V. Chandra and V. Ravishankar, Phys. Rev. D 84, 074013 (2011).

[123] S. Mitra and V. Chandra, Phys. Rev. D 96, 094003 (2017).

[124] X. Zhang and W. Chao, Nucl. Phys. A628, 161 (1998). 